\title{
Review
}

\section{Systematic Review of Discography as a Diagnostic Test for Spinal Pain: An Update}

Ricardo M. Buenaventura, MD1, Rinoo V. Shah, MD², Vikram Patel, MD³, Ramsin Benyamin, $\mathrm{MD}^{4}$, and Vijay Singh, $\mathrm{MD}^{5}$

From: 'Dayton Pain Med, Kettering, $\mathrm{OH} ;{ }^{2}$ Guthrie Clinic, Horseheads, NY; ${ }^{3}$ Loyola University Medical Center, Maywood, IL; ${ }^{4}$ Millennium Pain Center, Bloomington, IL; and ${ }^{5}$ Pain Diagnostics Associates, Niagara, WI. Dr. Buenaventura ${ }^{1}$ is Medica

Director, Dayton Pain Med, and Clinical Associated Professor,

Department of Surgery, Wright State University School of Medicine, Dayton, Ohio; Dr. Shah², Interventional Pain Management,

Department of Anesthesiology, Guthrie Clinic/Big Flats, Horseheads, NY; Dr. Patel 3 is Associate Professor of Anesthesiology, Director of Pain Fellowship Program, Department of

Anesthesiology, Loyola University

Medical Center, Maywood, IL; Dr.

Benyamin 4 is President, Millennium

Pain Center, Bloomington, IL; Dr.

Singh 5 is Medical Director, Pain

Diagnostic Associates, Niagara, WI. Address Correspondence: Ricardo M. Buenaventura, 3490 Far Hills Avenue, Suite 202, Kettering, OH 45429

E-mail: rbuena@yahoo.com Funding: None Conflict of Interest: None.

Free Full manuscript: www.painphysicianjournal.com
Background: The intervertebral disc has been implicated as an etiology of chronic spine pain based on clinical, basic science, and epidemiological research. There is currently no way to determine with absolute certainty whether or not the disc is a spinal pain generator. At our current level of understanding, discography is thought of as the best tool to evaluate disc-related pain.

Study Design: A systematic review.

Objective: To systematically assess the diagnostic accuracy of discography with respect to chronic spinal pain.

Methods: A systematic review of the literature was performed to assess the diagnostic accuracy of discography with respect to chronic spinal pain. Study inclusion/exclusion criteria were based on the modern practice of discography. Selected studies were then subjected to two rating instruments for diagnostic accuracy studies (AHRQ and QUADAS). Specific data were then culled from these studies and tabulated. Evidence was then classified into five levels: conclusive, strong, moderate, limited, or indeterminate.

Results: Evidence is strong for the diagnostic accuracy of discography as an imaging tool. Evidence is also strong for the ability of discography to evoke pain. There is strong evidence supporting the role of discography in identifying that subset of patients with lumbar discogenic pain. There is moderate evidence supporting the role of discography in identifying a subset of patients with cervical discogenic pain. There is limited evidence supporting the role of discography in identifying a subset of patients with thoracic discogenic pain.

Conclusion: Discography is a useful imaging and pain evaluation tool in identifying a subset of patients with chronic spinal pain secondary to intervertebral disc disorders.

Key words: Spinal pain, intervertebral disc, discography, pain generator, false-positives, diagnostic accuracy

Pain Physician 2007; 10:147-164 
D iscography was mainly used as an imaging tool in the past (1-11) and is considered to be superior to radiographs, myelography, and CT-scanning, in imaging intervertebral disc (IVD) morphology (12). Discography is comparable to MR imaging in detecting degenerative disc disease (DDD) $(4,13-16)$. The ability of discography to image the IVD has been validated by direct cadaveric and intraoperative examinations (17-21) and may be improved by using thin slice CT-scanning $(5,6,12,15,22-24)$. Some authors tout the merits of knowing about IVD morphology via discography (22).

Nonetheless, concerns about invasiveness, radiation exposure, infectious risk, and the routine availability of CT/MR imaging have minimized the role of discography as an imaging only tool (25). Furthermore, knowing IVD morphology, in isolation, may not be useful. DDD occurs due to age-related and genetic factors (26-30) and occurs with a similar frequency in symptomatic and asymptomatic patients (13).

DDD is not necessarily painful and even then, the pain may be variable (31). Although some authors (32) report increased pain with DDD, careful inspection of their data demonstrates that concordant pain occurs in a small fraction of patients. Conversely, DDD will not predict whether a disc is painful in a particular patient (33). In the case of annular disruption, the site of an annular tear may not correspond to the site of a patient's pain (29). Discograms do not image pain and hence, do not provide insight into which neural pathways mediate discogenic pain (34-52). IVD morphology should not change within a short interval, but discographic images may change after only 2 weeks. Technical errors account for only a small portion of these changes (53).

Whereas discographic imaging alone may not be useful, pain provocation in combination with real time imaging may be useful. Pain provocation, actually, dominates modern discographic practice and is used clinically (1,4-6,54-57). An integrative imaging approach, which incorporates provocative discography, may best select patients for intradiscal therapies (58-66).

So why perform another systematic review of discography? Systematic reviews of diagnostic test studies aim to identify and evaluate peer-reviewed medical research pertaining to diagnostic test accuracy. The quality assessment of diagnostic accuracy studies differs from that of standard intervention studies. To measure diagnostic accuracy, one must compare the test to the criterion standard. Since there is no crite- rion standard, a systematic review of the discography literature is challenging. A systematic review of studies evaluating existing studies that specifically examined the ability of discography, as a pain-provoking and imaging tool, to diagnose discogenic pain was first performed in 2005 (1). Since then, multiple publications have appeared in the literature and the controversy continues. Hence, this systematic review and update were performed to determine whether discography continues to be a useful test in the diagnostic evaluation of patients with chronic spinal pain.

\section{Methods}

This systematic review was updated based on a review performed in 2005 (1).

\section{Inclusion Criteria}

\section{Types of Studies}

Clinical studies in which discography was part of the study design and analysis were included. Randomized clinical trials were given preference over cohort and observational studies in the grading. Since the key elements of discography are pain provocation and imaging of the IVD, we specifically searched for contingency tables or data that compared pain provocation to IVD imaging.

\section{Types of Participants}

Asymptomatic volunteers or symptomatic patients with chronic spinal pain were included. Patients may or may not have undergone prior surgery.

\section{Types of Interventions}

Discography, whether alone or in combination with other diagnostic tests, should be described clearly. At a minimum, pain provocation and disc morphology should be reported. Post-discography image validation with $\mathrm{CT}$ scanning or MR imaging was not mandatory $(23,64)$.

\section{Exclusion Criteria}

Types of Studies

Non-clinical studies, technical papers, expert opinion, review articles, and single case reports were excluded. Also excluded were clinical studies that used discography to select patients for treatment, but did not analyze the discography data separately.

\section{Types of interventions}

If discography was not practiced in accordance with modern principles, the study was excluded $(1,4-$ 6,54-57,65): (1) the absence of ethical barriers, i.e., discography performed on unwilling or vulnerable participants; (2) the absence of real time pain/archi- 
tecture assessment or serial radiographs used instead of fluoroscopy; (3) the absence of systematic data reporting results that cannot be analyzed by the reader; (4) the assessment of pain provocation alone or morphology alone; and (5) the use of oil based or ionic, high-osmolar, water soluble dyes as these agents can be hazardous when used for spinal procedures. Types of Patients

Patients with chronic spinal pain due to a disc protrusion or verifiable non-discogenic etiology were excluded.

\section{Search Strategy}

Relevant clinical trials meeting the inclusion criteria for this review were identified in the following manner:

1. A computerized database search was performed of PUBMED (January 1, 2005 to November 30, 2006), CINAHL (Cumulative Index to Nursing \& Allied Health Literature), EMBASE, and Evidence Based Medicine Reviews (Cochrane Database and Cochrane Central Register of Controlled Trials). The following terms were used in the search: discogram, discography, intervertebral disc/diagnosis, intervertebral disc/injection, and intervertebral disc/pain. Boolean operators (AND/OR) and Boolean logic were used to optimize the search. Mesh headings were also used. A second iteration using the Mesh terms was used: Intervertebral Disk Displacement/radiography, Intervertebral Disk
Displacement/surgery, Injections, Spinal, and Sensitivity and Specificity, at the exclusion of the previous search strategy and at the exclusion of the terms, intrathecal and epidural. Limits (Adults, Human) were applied.

2. A review of the reference sections of selected articles was performed to identify other relevant studies.

3. Only English language articles were reviewed.

\section{Method of Review}

From the computerized database search, individual citations containing the author, title, keywords, and abstract were printed. Citations were reviewed for exclusion criteria and if none were found, the journal article was obtained. Five physicians reviewed these articles. The articles were then tabulated based on: 1) the methodological quality; 2 ) the study design; 3 ) the number of patients; 4) the discography technique; 5 ) the pain assessment; 6 ) the use of a control disc; 7) the use of an advanced imaging tool, post-discography; 8) the use of discography as the criterion standard to study another imaging tool; 9) the presence of contingency tables or data to assess sensitivity and specificity of the pain response for IVD morphology; and 10) the conclusions.

\section{Methodological Quality}

The quality of each individual article was evaluated by the AHRQ (Table 1) and QUADAS (Table 2)

Table 1. Domains and elements for diagnostic studies developed by the Agency for Healthcare Research and Quality (AHRQ).

\begin{tabular}{|c|c|}
\hline Study Population & $\begin{array}{l}\text { - Subjects similar to populations in which the test would be used and with a similar spectrum of } \\
\text { disease }\end{array}$ \\
\hline Adequate Description of Test & - Details of test and its administration sufficient to allow for replication of study \\
\hline Appropriate Reference Standard & $\begin{array}{l}\text { - Appropriate reference standard (gold standard) used for comparison } \\
\text { - Reference standard reproducible }\end{array}$ \\
\hline $\begin{array}{l}\text { Blinded Comparison of } \\
\text { Test and Reference }\end{array}$ & $\begin{array}{l}\text { - Evaluation of test without knowledge of disease status, if possible } \\
\text { - Independent, blind interpretation of test and reference }\end{array}$ \\
\hline Avoidance of Verification Bias & - Decision to perform reference standard not dependent on results of test under study \\
\hline \multicolumn{2}{|c|}{$\begin{array}{l}\text { "Key domains are in italics. } \\
{ }^{*} \text { Elements appearing in italics are those with an empirical basis. Elements appearing in bold are those considered essential to give a system a } \\
\text { Yes rating for the domain. }\end{array}$} \\
\hline Adapted from ref 67 & \\
\hline
\end{tabular}


Table 2. Items utilized for assessment of quality of individual articles of diagnostic studies by QUADAS tool (68)

\begin{tabular}{|c|c|}
\hline \multicolumn{2}{|c|}{ Item } \\
\hline 1. & Was the spectrum of patients representative of the patients who will receive the test in practice? \\
\hline 2. & Were selection criteria clearly described? \\
\hline 3. & Is the reference standard likely to correctly classify the target condition? \\
\hline 4. & $\begin{array}{l}\text { Is the time period between reference standard and index test short enough to be reasonably sure that the target condition did } \\
\text { not change between the two tests? }\end{array}$ \\
\hline 5. & Did the whole sample or a random selection of the sample receive verification using a reference standard of diagnosis? \\
\hline 6. & Did patients receive the same reference standard regardless of the index test result? \\
\hline 7. & Was the reference standard independent of the index test (i.e. the index test did not form past of the reference standard)? \\
\hline 8. & Was the execution of the index test described in sufficient detail to permit replication of the test? \\
\hline 9. & Was the execution of the reference standard described in sufficient detail to permit its replication? \\
\hline 10. & Were the index test results interpreted without knowledge of the results of the reference standard? \\
\hline 11. & Were the reference standard results interpreted without knowledge of the results of the index test? \\
\hline 12. & Were the same clinical data available when test results were interpreted as would be available when the test is used in practice? \\
\hline 13. & Were uninterpretable/intermediate test results reported? \\
\hline 14. & Were withdrawals from the study explained? \\
\hline
\end{tabular}

rating scales (67-69). The AHRQ (5 points) and the QUADAS (14 points) are diagnostic study scoring instruments. Their parameters are shown in Tables 1 and 2. We assigned pain provocation to be the index test and IVD imaging to be the gold standard. As described earlier, the validity of discography as an imaging tool has been investigated. The imaging information is important since treating an anatomically normal disc, irrespective of its ability to cause pain, seems unethical $(13,70)$.

Studies were then scored according to the AHRQ and QUADAS rating instruments, with the foregoing assumptions in mind. For inclusion, the studies had to meet at least $50 \%$ of the points for each scale (i.e., 3 of 5 for the AHRQ or 7 of 14 for the QUADAS); studies were excluded if their scores were 2 of 5 for the AHRQ and 6 of 14 for the QUADAS.

\section{Strength of Evidence}

The level of evidence was evaluated as shown in Table 3.

\section{RESULTS}

\section{Literature Search}

For the period of January 1, 2005, to November 30,2006 , the database search yielded 56 articles. 35 were immediately excluded, by title alone. 21 abstracts were further reviewed. 16 were relevant to the study question and 16 full text journal articles were requested and reviewed. 6 articles failed to meet the inclusion criteria. 10 articles met the inclusion criteria. After scoring with the AHRQ and QUADAS rating instruments, all 10 articles qualified (71-80). This current systematic review addresses these 10 articles. In combination, however, with the 71 articles $(12,18,21$ $25,31,32,66,70,81-140)$ identified in our prior systematic review (1), the total number of articles that have met the threshold for inclusion is 81 (Fig. 1). The original 71 articles are included in the references and the original data detailing the articles and their scoring by AHRQ and QUADAS criteria along with new data are illustrated in Tables 4-6. 
Table 3. Designation of levels of evidence(2).

\begin{tabular}{|l|l||}
\hline Level I & $\begin{array}{l}\text { Conclusive: Research-based evidence with multiple relevant and high-quality scientific studies or consistent reviews of meta- } \\
\text { analyses. }\end{array}$ \\
\hline Level II & $\begin{array}{l}\text { Strong: Research-based evidence from at least 1 properly designed randomized, controlled trial; or research-based evidence } \\
\text { from multiple properly designed studies of smaller size; or multiple low quality trials. }\end{array}$ \\
\hline Level III & $\begin{array}{l}\text { Moderate: } \\
\text { a) Evidence obtained from well-designed pseudorandomized controlled trials (alternate allocation or some other method); } \\
\text { b) evidence obtained from comparative studies with concurrent controls and allocation not randomized (cohort studies, } \\
\text { case-controlled studies, or interrupted time series with a control group); } \\
\text { c) evidence obtained from comparative studies with historical control, two or more single-arm studies, or interrupted time } \\
\text { series without a parallel control group. }\end{array}$ \\
\hline Level IV & $\begin{array}{l}\text { Limited: Evidence from well-designed nonexperimental studies from more than 1 center or research group; or conflicting } \\
\text { evidence with inconsistent findings in multiple trials. }\end{array}$ \\
\hline Level V & $\begin{array}{l}\text { Indeterminate: Opinions of respected authorities, based on clinical evidence, descriptive studies, or reports of expert } \\
\text { committees. }\end{array}$ \\
\hline
\end{tabular}

\section{Methodological Quality}

In combination with our prior systematic review (1), there was: 1) 1 prospective, randomized and controlled study; 2) 2 randomized and prospective studies; 3) 7 prospective, controlled studies; 4) 1 paper that was prospective and case controlled; 5) 1 prospective, blinded study; 6) 39 prospective studies; 7) 1 paper that was both retrospective and prospective; 8) 28 retrospective studies; 9) 1 retrospective and blinded study; and 10) no double blinded, randomized controlled studies. Overall, there were 10 papers pertaining to cervical discography $(66,82,94,108,109,115,12$ $2,123,127,139)$, two pertaining to thoracic discography $(124,137)$, and 69 pertaining to lumbar discography $\quad(12,18,21,22-25,31,32,70-81,83-93,95-107,110-$ $114,116-121,125,126,128-136,138,140)$. Data from the 10 new articles are incorporated with old data in Tables 4-6. The 10 articles identified, subsequent to our last systematic review (1), are summarized below. The 71 other articles have already been discussed in our previous systematic review (1). For purposes of brevity and due to ease of access, this information has not been presented again.

Blankenbaker et al (71) studied 16 patients who had concordant pain at discography. These patients were then recruited for a study evaluating axial rotation of the involved lumbar spinal motion segments.
There were placed on a special CT scanner table that could rotate the pelvis $8^{\circ}$ clockwise and counterclockwise with respect to the thorax. They found that concordant pain at discography predicted increased axial rotation at the lumbar disc level.

Carragee et al (72) performed a prospective, longitudinal study of 100 subjects with high risk factors for serious low back pain (LBP) as determined by structural and psychosocial characteristics. All subjects had known risk factors for degenerative lumbar disc disease and a history of mild, persistent but nondisabling LBP. All subjects were evaluated for lumbar spinal pathology by physical examination, plain radiography, and MRI and also underwent psychological testing. A subgroup of psychologically normal patients also underwent provocative lumbar discography. They were followed for 6 month intervals for 5 years and assessed for disability due to LBP. The development of serious LBP disability was strongly associated with baseline psychosocial variables. Modic changes of the vertebral endplate as seen on MRI were weakly associated with any adverse outcome. A positive provocative discogram at baseline did not predict any future adverse outcome.

In a retrospective data review of positive (painful) disc injections at low pressures among 69 subjects with and without chronic LBP, Carragee et al (73) found 


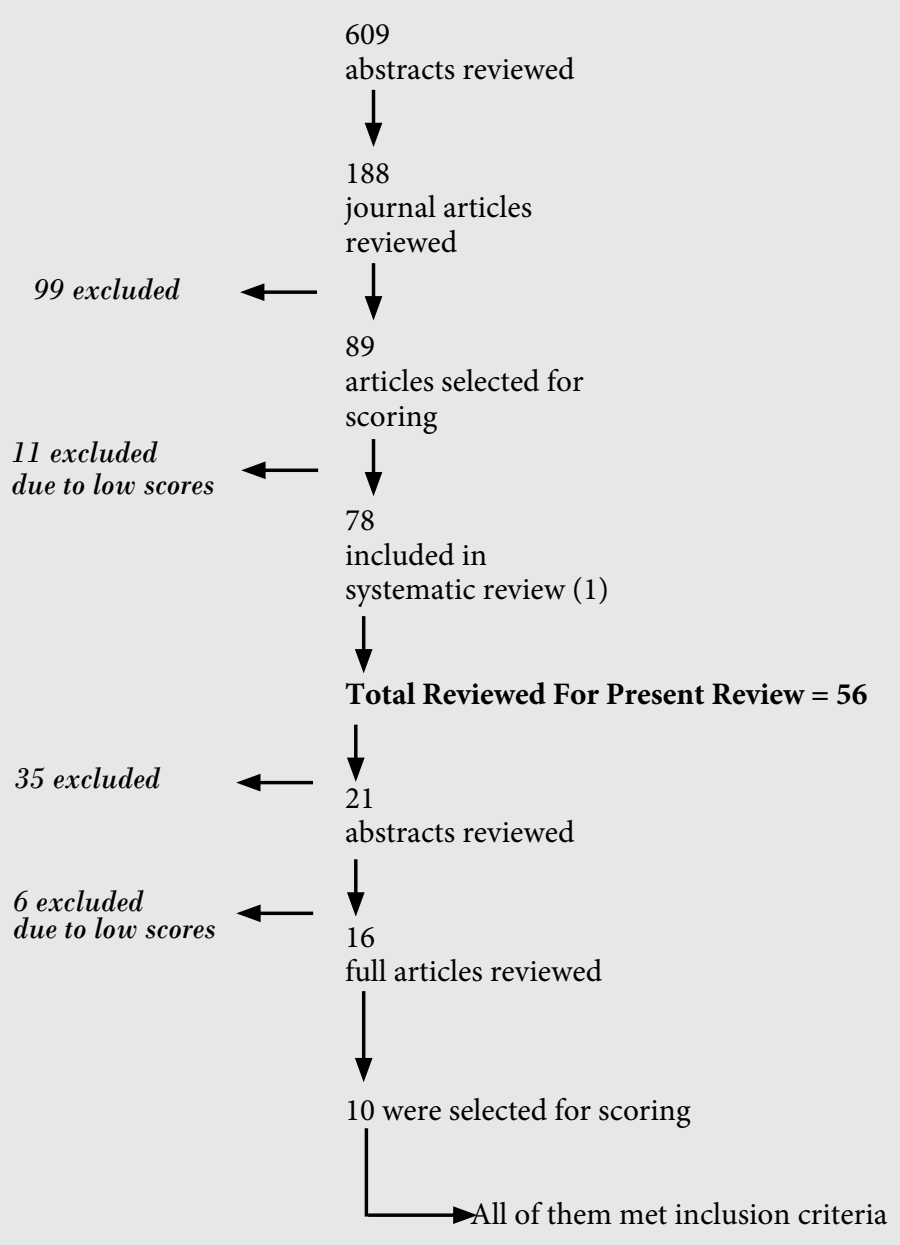

Fig.1. Systematic review flow sheet, modified from and combined with Shah et al (1).

that the rate of low pressure ( $<22$ psi) painful injection in subjects without significant chronic LBP is approximately $25 \%$. This correlated with both anatomic and psychosocial factors. They felt that $25 \%$ positive painful discs in asymptomatic individuals may represent an unacceptable risk of false positive results.

Carragee et al (74) attempted to test the hypothesis that positive discography accurately identifies a LBP illness due to a primary discogenic lesion as determined by clinical cure with successful arthrodesis in a best case clinical setting. They compared a group of patients with LBP and a positive single-level low-pressure provocative discogram to another group with single level unstable Grade I-II isthmic spondylolisthesis either at L4-5 or L5-
S1. Both groups later underwent spinal fusion of the involved level. The subjects were followed for 2 years. Several criteria were used to assess successful outcomes. $72 \%$ of the spondylolisthesis group versus $27 \%$ of the discogenic pain group met the highly effective success criteria. The authors felt that positive discography was not highly predictive in identifying bona fide isolated intradiscal lesions primarily causing LBP illness.

Derby et al (75) performed pressure-controlled discography in 13 asymptomatic volunteers (43 discs). The purpose of this prospective study was to determine the prevalence of positive responses to lumbar discography in asymptomatic volunteers. $56 \%$ of the injected discs were not painful. $44 \%$ were painful 
Table 4. Characteristics of articles included in review for cervical discography

\begin{tabular}{|c|c|c|c|c|c|c|c|c|c|c|}
\hline Study & $\underset{2}{\mathbb{Z}}$ & 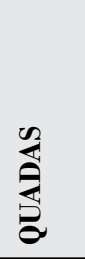 & 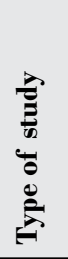 & 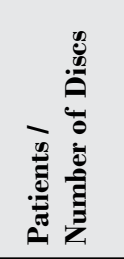 & 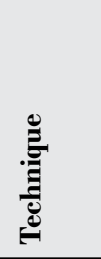 & 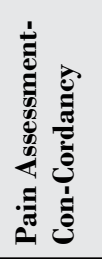 & 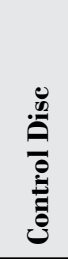 & 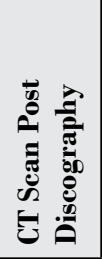 & 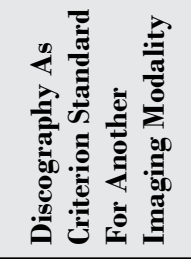 & 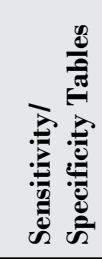 \\
\hline Grubb and Kelly (66) & $4 / 5$ & $9 / 14$ & $\mathrm{R}$ & 173 & $\mathrm{RAO}$ & $\mathrm{Y}$ & $\mathrm{Y}$ & $\mathrm{Y}$ & $\mathrm{N}$ & $\mathrm{Y}$ \\
\hline Bogduk and Aprill (82) & $4 / 5$ & $11 / 14$ & $\mathrm{R}$ & 56 & RAO & $\mathrm{Y}$ & $\mathrm{N}$ & $\mathrm{N}$ & $\mathrm{N}$ & $\mathrm{N}$ \\
\hline $\begin{array}{l}\text { Connor and Darden } \\
(94)\end{array}$ & $3 / 5$ & $10 / 14$ & $\mathrm{R}$ & 31 & RAO & $\mathrm{Y}$ & $\mathrm{U}$ & $\mathrm{N}$ & $\mathrm{N}$ & $\mathrm{N}$ \\
\hline Motimaya et al (108) & $3 / 5$ & $7 / 14$ & $\mathrm{R}$ & $16 / 46$ & LAO & $\mathrm{Y}$ & $\mathrm{U}$ & $\mathrm{N}$ & $\mathrm{N}$ & $\mathrm{N}$ \\
\hline Ohnmeiss et al (109) & $4 / 5$ & $11 / 14$ & $\mathrm{R}$ & $161 / 269$ & $\mathrm{E}$ & $\mathrm{Y}$ & $\mathrm{N}$ & $\mathrm{Y}$ & $\mathrm{N}$ & $\mathrm{N}$ \\
\hline $\begin{array}{l}\text { Parfenchuck and } \\
\text { Janssen (115) }\end{array}$ & $4 / 5$ & $11 / 14$ & $\mathrm{P}$ & 52 & UAO & $\mathrm{Y}$ & $\mathrm{U}$ & $\mathrm{Y}$ & $\mathrm{N}$ & $\mathrm{Y}$ \\
\hline Schellhas et al (122) & $3 / 5$ & $11 / 14$ & $\mathrm{P}$ & $\begin{array}{l}10 \mathrm{Asx} / \\
10 \mathrm{Sx}\end{array}$ & $\mathrm{RAO}$ & $\mathrm{Y}$ & Y & $\mathrm{Y} / \mathrm{N}$ & $\mathrm{N}$ & $\mathrm{Y}$ \\
\hline Schellhas et al (123) & $4 / 5$ & $11 / 14$ & $\mathrm{R}, \mathrm{P}$ & 40 & RAO & $\mathrm{Y}$ & $\mathrm{Y}$ & $\mathrm{N}$ & $\mathrm{N}$ & $\mathrm{N}$ \\
\hline $\begin{array}{l}\text { Siebenrock and Aebi } \\
\text { (127) }\end{array}$ & $4 / 5$ & $11 / 14$ & $\mathrm{R}$ & $27 / 39$ & L/RAO & $\mathrm{Y}$ & $\mathrm{N}$ & $\mathrm{N}$ & $\mathrm{N}$ & $\mathrm{N}$ \\
\hline Zheng et al (139) & $4 / 5$ & $11 / 14$ & $\mathrm{R}$ & $55 / 161$ & RAO & $\mathrm{Y}$ & $\mathrm{U}$ & $\mathrm{Y}$ & $\mathrm{Y}$ & Y \\
\hline
\end{tabular}

$R=$ retrospective; $P=$ prospective $Y=$ yes; $N=$ No; $E=$ extrapedicular $L=$ left $U=$ unknown; $U A O=$ side unknown, anterior oblique; $R A O=$ right anterior oblique; $A s x=$ asymptomatic; $S X=$ symptomatic

Table 5. Characteristics of articles included in review for thoracic discography

\begin{tabular}{|c|c|c|c|c|c|c|c|c|c|c|}
\hline Study & 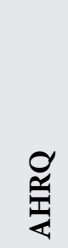 & 蛋 & 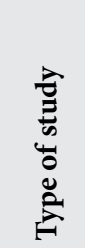 & 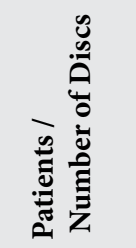 & 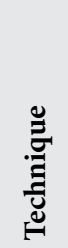 & 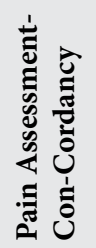 & 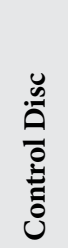 & 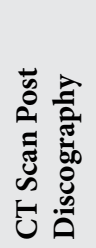 & 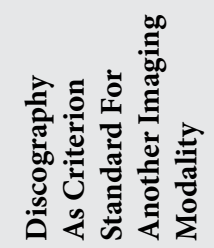 & 总 \\
\hline Wood et al (137) & $4 / 5$ & $11 / 14$ & $\mathrm{P}, \mathrm{CC}$ & $\begin{array}{l}10 \text { Asx } / 10 \\
\text { Sx }\end{array}$ & $\mathrm{E}$ & $\mathrm{Y}$ & $\mathrm{Y}$ & $\mathrm{N}$ & $\mathrm{Y}$ & $\mathrm{Y}$ \\
\hline Schellhas et al (124) & $4 / 5$ & $11 / 14$ & $\mathrm{R}$ & 100 & $\mathrm{E}$ & $\mathrm{Y}$ & $\mathrm{Y}$ & $\mathrm{Y} / \mathrm{N}$ & $\mathrm{N}$ & $\mathrm{N}$ \\
\hline
\end{tabular}

$R=$ retrospective $P=$ prospective CC $=$ case controlled $Y=$ yes $N=$ No; $E=$ extrapedicular $;$ As $x=$ asymptomatic $; S X=$ symptomatic 
Pain Physician: January 2007:10:147-164

Table 6. Characteristics of articles included in review for lumbar discography.

\begin{tabular}{|c|c|c|c|c|c|c|c|c|c|c|}
\hline$\stackrel{\vec{E}}{E}$ & 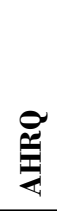 & 岕 & 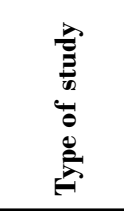 & 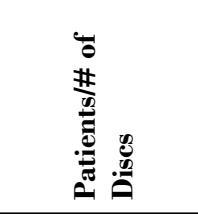 & 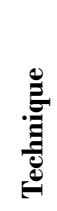 & 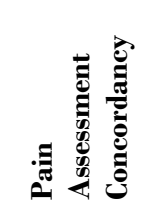 & : & 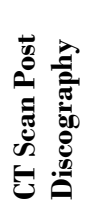 & 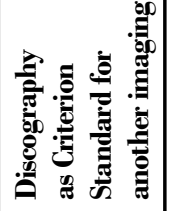 & 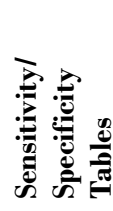 \\
\hline Abdelwahab et al (18) & $3 / 5$ & $7 / 14$ & $\mathrm{R}$ & 15 & $\mathrm{E} / \mathrm{I}$ & $\mathrm{Y}$ & $\mathrm{N}$ & $\mathrm{N}$ & $\mathrm{Y}$ & $\mathrm{N}$ \\
\hline Antti-Poika et al (12) & $4 / 5$ & $11 / 14$ & $\mathrm{P}$ & 100 & $\mathrm{E}$ & $\mathrm{Y}$ & $\mathrm{U}$ & $\mathrm{Y}$ & $\mathrm{N}$ & $\mathrm{Y}$ \\
\hline Aprill and Bogduk (81) & $4 / 5$ & $11 / 14$ & $\mathrm{P}$ & 41 & $\mathrm{E}$ & $\mathrm{Y}$ & $\mathrm{Y}$ & $\mathrm{Y}$ & $\mathrm{Y}$ & $\mathrm{Y}$ \\
\hline Bernard (22) & $4 / 5$ & $11 / 14$ & $\mathrm{P}$ & 250 & $\mathrm{E}$ & $\mathrm{Y}$ & $\mathrm{N}$ & $\mathrm{Y}$ & $\mathrm{Y}$ & $\mathrm{N}$ \\
\hline Blankenbaker et al (71) & $3 / 5$ & $7 / 14$ & $\mathrm{P}$ & $16 \mathrm{pt} / 94 \mathrm{~d}$ & $\mathrm{E}$ & $\mathrm{Y}$ & $\mathrm{N}$ & $\mathrm{Y}$ & $\mathrm{Y}$ & $\mathrm{N}$ \\
\hline Block et al (25) & $4 / 5$ & $11 / 14$ & $\mathrm{P}$ & 72 & $\mathrm{E}$ & $\mathrm{Y}$ & $\mathrm{Y}$ & $\mathrm{Y}$ & $\mathrm{Y}$ & $\mathrm{Y}$ \\
\hline Braithwaite et al (83) & $4 / 5$ & $11 / 14$ & $\mathrm{R}$ & 58 & $\mathrm{E}$ & $\mathrm{Y}$ & $\mathrm{Y}$ & $\mathrm{Y}$ & $\mathrm{Y}$ & $\mathrm{Y}$ \\
\hline Carragee et al (84) & $4 / 5$ & $12 / 14$ & $\mathrm{P} / \mathrm{C}$ & 26 Asx / 6 Sx & $\mathrm{E}$ & $\begin{array}{c}\text { N-intensity } \\
\text { only }\end{array}$ & $\mathrm{N}$ & $\mathrm{N}$ & $\mathrm{N}$ & $\mathrm{N}$ \\
\hline Carragee et al (85) & $5 / 5$ & $12 / 14$ & $\mathrm{P} / \mathrm{C}$ & $\begin{array}{c}46 \mathrm{disco} / 49 \text { no } \\
\text { disco }\end{array}$ & $\mathrm{E}$ & $\begin{array}{c}\mathrm{N} \text {-intensity } \\
\text { only }\end{array}$ & $\mathrm{N}$ & $\mathrm{N}$ & $\mathrm{N}$ & $\mathrm{N}$ \\
\hline Carragee et al (86) & $4 / 5$ & $11 / 14$ & $\mathrm{P} / \mathrm{C}$ & $\begin{array}{c}25 \text { mild LBP52 } \\
\text { severe LBP }\end{array}$ & $\mathrm{E}$ & $\mathrm{Y}$ & $\mathrm{Y}$ & $\mathrm{N}$ & $\mathrm{N}$ & $\mathrm{Y}$ \\
\hline Carragee et al (87) & $4 / 5$ & $11 / 14$ & $\mathrm{P} / \mathrm{RA}, \mathrm{C}$ & 20 Asx -27 Sx & $\mathrm{E}$ & $\mathrm{Y}$ & $\mathrm{Y}$ & $\mathrm{N}$ & $\mathrm{N}$ & $\mathrm{Y}$ \\
\hline Carragee et al (88) & $3 / 5$ & $10 / 14$ & $\mathrm{P}$ & $\begin{array}{c}8 \text { Asx-iliac crest } \\
\text { harvest }\end{array}$ & $\mathrm{E}$ & $\mathrm{Y}$ & $\mathrm{N}$ & $\mathrm{N}$ & $\mathrm{N}$ & $\mathrm{Y}$ \\
\hline Carragee et al (89) & $3 / 5$ & $10 / 14$ & $\mathrm{P}$ & 26 Asx pts. & $\mathrm{E}$ & $\mathrm{Y}$ & $\mathrm{N}$ & $\mathrm{N}$ & $\mathrm{N}$ & $\mathrm{Y}$ \\
\hline Carragee et al (90) & $4 / 5$ & $11 / 14$ & $\mathrm{P} / \mathrm{C}$ & $42 \mathrm{Sx} ; 52$ Asx & $\mathrm{E}$ & $\mathrm{Y}$ & $\mathrm{Y}$ & $\begin{array}{l}\text { Y } 31 / \\
\text { N } 52\end{array}$ & $\mathrm{~N}$ & $\mathrm{Y}$ \\
\hline Carragee et al (72) & $3 / 5$ & $9 / 14$ & $\mathrm{P}$ & $\begin{array}{c}25 \text { pt disco / } 75 \\
\text { pt no disco; \# d } \\
\text { not recorded }\end{array}$ & $\mathrm{E}$ & $\mathrm{Y}$ & $\mathrm{Y}$ & $\mathrm{N}$ & $\mathrm{N}$ & $\mathrm{Y}$ \\
\hline Carragee et al (73) & $3 / 5$ & $8 / 14$ & $\mathrm{R}, \mathrm{B}$ & $\begin{array}{c}69 \text { pt; but } 20 \mathrm{pt} \\
\text { w/prev. surg.; \# d } \\
\text { not recorded }\end{array}$ & $\mathrm{E}$ & $\mathrm{Y}$ & $\mathrm{Y}$ & $\mathrm{N}$ & $\mathrm{N}$ & $\mathrm{Y}$ \\
\hline Carragee et al (74) & $3 / 5$ & $8 / 14$ & $\mathrm{P}$ & $\begin{array}{c}32 \mathrm{pt} \text { disco/ } 34 \\
\text { pt no disco. \# d } \\
\text { not recorded }\end{array}$ & $\mathrm{E}$ & $\mathrm{Y}$ & $\mathrm{Y}$ & $\mathrm{N}$ & $\mathrm{N}$ & $\mathrm{Y}$ \\
\hline Cohen et al (91) & $4 / 5$ & $10 / 14$ & $\mathrm{R}$ & 127 & $\mathrm{E}$ & $\mathrm{Y}$ & $\mathrm{Y}$ & $\mathrm{N}$ & $\mathrm{N}$ & $\mathrm{N}$ \\
\hline Colhoun et al (92) & $3 / 5$ & $11 / 14$ & $\mathrm{R}$ & 195 & $\mathrm{E}$ & $\mathrm{Y}$ & $\mathrm{U}$ & $\mathrm{N}$ & $\mathrm{N}$ & $\mathrm{N}$ \\
\hline Collins et al (93) & $4 / 5$ & $11 / 14$ & $\mathrm{P}$ & 29 & $\mathrm{E}$ & $\mathrm{Y}$ & $\mathrm{U}$ & $\mathrm{N}$ & $\mathrm{Y}$ & $\mathrm{N}$ \\
\hline Derby et al (95) & $4 / 5$ & $11 / 14$ & $\mathrm{R}$ & 146 & $\mathrm{E}$ & $\mathrm{Y}$ & $\mathrm{U}$ & $\mathrm{U}$ & $\mathrm{N}$ & $\mathrm{N}$ \\
\hline Derby et al (75) & $4 / 5$ & $11 / 14$ & $\mathrm{P}, \mathrm{C}$ & $13 \mathrm{pt} / 43 \mathrm{~d}$ & $\mathrm{E}$ & $\mathrm{Y}$ & $\mathrm{N}$ & $\mathrm{N}$ & $\mathrm{N}$ & $\mathrm{Y}$ \\
\hline Derby et al (76) & $3 / 5$ & $11 / 14$ & $\mathrm{P}$ & $106 \mathrm{pt} / 337 \mathrm{~d}$ & $\mathrm{E}$ & $\mathrm{Y}$ & $\mathrm{N}$ & $\mathrm{Y}$ & $\mathrm{N}$ & $\mathrm{Y}$ \\
\hline Derby et al (77) & $4 / 5$ & $11 / 14$ & $\mathrm{P}, \mathrm{C}$ & $86 \mathrm{pt} / 279 \mathrm{~d}$ & $\mathrm{E}$ & $\mathrm{Y}$ & $\mathrm{N}$ & $\mathrm{Y}$ & $\mathrm{N}$ & $\mathrm{Y}$ \\
\hline Donelson et al (96) & $4 / 5$ & $11 / 14$ & $\mathrm{P}$ & 63 & $\mathrm{E}$ & $\mathrm{Y}$ & $\mathrm{Y}$ & $\mathrm{Y}$ & $\mathrm{Y}$ & $\mathrm{N}$ \\
\hline Gill and Blumenthal (70) & $3 / 5$ & $9 / 14$ & $\mathrm{R}$ & 53 & $\mathrm{U}$ & $\mathrm{Y}$ & $\mathrm{U}$ & $\mathrm{N}$ & $\mathrm{N}$ & $\mathrm{N}$ \\
\hline Greenspan et al (97) & $4 / 5$ & $11 / 14$ & $\mathrm{P}$ & $32 / 78$ & $\mathrm{I}$ & $\mathrm{Y}$ & $\mathrm{U}$ & $\mathrm{Y}$ & $\mathrm{N}$ & $\mathrm{Y}$ \\
\hline Heggeness et al (98) & $3 / 5$ & $8 / 14$ & $\mathrm{R}$ & $83 / 238$ & $\mathrm{U}$ & $\mathrm{Y}$ & $\mathrm{U}$ & $\mathrm{N}$ & $\mathrm{N}$ & $\mathrm{Y}$ \\
\hline Horton and Daftari (99) & $4 / 5$ & $11 / 14$ & $\mathrm{P}$ & $25 / 63$ & $\mathrm{E}$ & $\mathrm{Y}$ & $\mathrm{U}$ & $\mathrm{N}$ & $\mathrm{Y}$ & $\mathrm{N}$ \\
\hline Ito et al (100) & $5 / 5$ & $12 / 14$ & $\mathrm{P}$ & $39 / 101$ & $\mathrm{E}$ & $\mathrm{Y}$ & $\mathrm{U}$ & $\mathrm{Y}$ & $\mathrm{Y}$ & $\mathrm{Y}$ \\
\hline Lam et al (101) & $5 / 5$ & $11 / 14$ & $\mathrm{P}, \mathrm{B}$ & 73 & $\mathrm{E}$ & $\mathrm{Y}$ & $\mathrm{U}$ & $\mathrm{N}$ & $\mathrm{Y}$ & $\mathrm{Y}$ \\
\hline Lim et al (78) & $4 / 5$ & $11 / 14$ & $\mathrm{P}$ & $47 \mathrm{pt} / 97 \mathrm{~d}$ & $\mathrm{E}$ & $\mathrm{Y}$ & $\mathrm{N}$ & $\mathrm{Y}$ & $\mathrm{Y}$ & $\mathrm{Y}$ \\
\hline Madan et al (102) & $3 / 5$ & $8 / 14$ & $\mathrm{P}, \mathrm{C}$ & 73 & $\mathrm{E}$ & $\mathrm{Y}$ & $\mathrm{U}$ & $\mathrm{N}$ & $\mathrm{N}$ & $\mathrm{N}$ \\
\hline
\end{tabular}


A Systematic Review of Discography as a Diagnostic Test for Spinal Pain

Table 6 Continued. Characteristics of articles included in review for lumbar discography.

\begin{tabular}{|c|c|c|c|c|c|c|c|c|c|c|}
\hline$\frac{\vec{E}}{\vec{E}}$ & 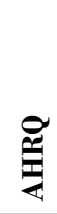 & 胥 & 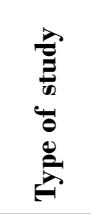 & 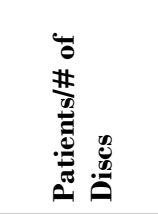 & 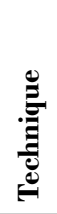 & 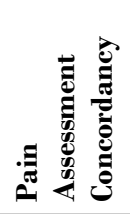 & $\frac{\dot{0}}{\mathscr{H}^{\circ}}$ & 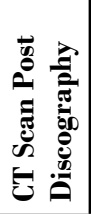 & 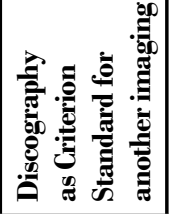 & 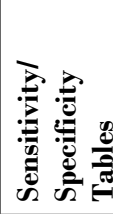 \\
\hline Maezawa et al (103) & $4 / 5$ & $10 / 14$ & $\mathrm{R}$ & $523 / 1477$ & $\mathrm{E}$ & $\mathrm{Y}$ & $\mathrm{U}$ & $\mathrm{Y}$ & $\mathrm{N}$ & $\mathrm{Y}$ \\
\hline Manchikanti et al (104) & $3 / 5$ & $10 / 14$ & P, RA & 50 & $\mathrm{E}$ & $\mathrm{Y}$ & $\mathrm{U}$ & $\mathrm{N}$ & $\mathrm{N}$ & $\mathrm{N}$ \\
\hline Manchikanti et al (105) & $3 / 5$ & $8 / 14$ & P, RA & 120 & $\mathrm{E}$ & $\mathrm{Y}$ & $\mathrm{Y}$ & $\mathrm{N}$ & $\mathrm{N}$ & $\mathrm{N}$ \\
\hline McCutcheon et al (24) & $4 / 5$ & $11 / 14$ & $\mathrm{R}$ & 22 & $\mathrm{E} / \mathrm{I}$ & $\mathrm{Y}$ & $\mathrm{U}$ & $\mathrm{Y}$ & $\mathrm{Y}$ & $\mathrm{N}$ \\
\hline McFadden (106) & $4 / 5$ & $11 / 14$ & $\mathrm{P}$ & 200 & $\mathrm{E}$ & $\mathrm{Y}$ & $\mathrm{U}$ & $\mathrm{Y}$ & $\mathrm{N}$ & $\mathrm{N}$ \\
\hline Milette et al (21) & $4 / 5$ & $12 / 14$ & $\mathrm{R}$ & 58 & I & $\mathrm{Y}$ & $\mathrm{U}$ & $\mathrm{Y} / \mathrm{N}$ & $\mathrm{Y}$ & $\mathrm{Y}$ \\
\hline Milette et al (107) & $4 / 5$ & $11 / 14$ & $\mathrm{P}$ & 17 & I & $\mathrm{Y}$ & $\mathrm{U}$ & $\mathrm{N}$ & $\mathrm{N}$ & $\mathrm{N}$ \\
\hline Moneta et al (31) & $4 / 5$ & $9 / 14$ & $\mathrm{R}$ & $306 / 833$ & $\mathrm{E}$ & $\mathrm{Y}$ & $\mathrm{U}$ & $\mathrm{Y}$ & $\mathrm{Y}$ & $\mathrm{Y}$ \\
\hline Ohnmeiss et al (110) & $4 / 5$ & $11 / 14$ & $\mathrm{P}$ & 170 & $E$ & $\mathrm{Y}$ & $\mathrm{U}$ & $\mathrm{Y}$ & $\mathrm{N}$ & $\mathrm{N}$ \\
\hline Ohnmeiss et al (111) & $4 / 5$ & $11 / 14$ & $\mathrm{P}$ & 187 & $\mathrm{E}$ & $\mathrm{Y}$ & $\mathrm{U}$ & $\mathrm{Y}$ & $\mathrm{N}$ & $\mathrm{Y}$ \\
\hline Ohnmeiss et al (112) & $4 / 5$ & $11 / 14$ & $\mathrm{P}$ & 187 & $\mathrm{E}$ & $\mathrm{Y}$ & $\mathrm{U}$ & $\mathrm{Y}$ & $\mathrm{N}$ & $\mathrm{Y}$ \\
\hline $\begin{array}{l}\text { O’Neill and Kurgansky } \\
\text { (113) }\end{array}$ & $4 / 5$ & $11 / 14$ & $\mathrm{R}$ & $253 / 838$ & E & $\mathrm{Y}$ & $\mathrm{Y}$ & $\mathrm{N}$ & $\mathrm{N}$ & $\mathrm{N}$ \\
\hline Osti and Fraser (114) & $4 / 5$ & $11 / 14$ & $\mathrm{P}$ & $33 / 114$ & $\mathrm{E}$ & $\mathrm{Y}$ & $\mathrm{U}$ & $\mathrm{N}$ & $\mathrm{Y}$ & $\mathrm{Y}$ \\
\hline Parker et al (116) & $1 / 5$ & $7 / 14$ & $\mathrm{P}$ & 23 & $\mathrm{E}$ & $\mathrm{Y}$ & $\mathrm{Y}$ & $\mathrm{N}$ & $\mathrm{Y}$ & $\mathrm{N}$ \\
\hline Peng et al (79) & $3 / 5$ & $8 / 14$ & $\mathrm{P}$ & $52 \mathrm{pt} / 142 \mathrm{~d}$ & $\mathrm{E}$ & $\mathrm{Y}$ & $\mathrm{N}$ & $\mathrm{Y}$ & $\mathrm{Y}$ & $\mathrm{N}$ \\
\hline Ricketson et al (117) & $4 / 5$ & $11 / 14$ & $\mathrm{P}$ & 29 & $\mathrm{E}$ & $\mathrm{Y}$ & $\mathrm{U}$ & $\mathrm{Y}$ & $\mathrm{Y}$ & $\mathrm{Y}$ \\
\hline Sachs et al (23) & $4 / 5$ & $11 / 14$ & $\mathrm{P}$ & 59 & $\mathrm{E}$ & $\mathrm{Y}$ & $\mathrm{U}$ & $\mathrm{Y}$ & $\mathrm{N}$ & $\mathrm{Y}$ \\
\hline Saiffudin et al (118) & $4 / 5$ & $11 / 14$ & $\mathrm{R}$ & $58 / 152$ & $\mathrm{E}$ & $\mathrm{Y}$ & $\mathrm{U}$ & $\mathrm{N}$ & $\mathrm{Y}$ & $\mathrm{Y}$ \\
\hline Saiffudin et al (119) & $4 / 5$ & $11 / 14$ & $\mathrm{R}$ & $99 / 260$ & $\mathrm{E}$ & $\mathrm{Y}$ & $\mathrm{U}$ & $\mathrm{N}$ & $\mathrm{N}$ & $\mathrm{Y}$ \\
\hline Schechter et al (120) & $3 / 5$ & $8 / 14$ & $\mathrm{R}$ & $20 / 32$ & $\mathrm{U}$ & $\mathrm{Y}$ & $\mathrm{N}$ & $\mathrm{N}$ & $\mathrm{N}$ & $\mathrm{N}$ \\
\hline Schellhas et al (121) & $4 / 5$ & $11 / 14$ & $\mathrm{R}$ & 63 & $\mathrm{E} / \mathrm{I}$ & $\mathrm{Y}$ & $\mathrm{Y}$ & $\mathrm{Y} / \mathrm{N}$ & $\bar{Y}$ & $\mathrm{~N}$ \\
\hline Schwarzer et al (125) & $4 / 5$ & $11 / 14$ & $\mathrm{P}$ & $92 / 255$ & E & $\mathrm{Y}$ & $\mathrm{Y}$ & $\mathrm{Y}$ & $\mathrm{N}$ & $\mathrm{Y}$ \\
\hline Schwarzer et al (126) & $4 / 5$ & $11 / 14$ & $\mathrm{P}$ & 92 & $\mathrm{E}$ & $\mathrm{Y}$ & $\mathrm{Y}$ & $\mathrm{Y}$ & $\mathrm{N}$ & $\mathrm{N}$ \\
\hline Shin et al (80) & $4 / 5$ & $11 / 14$ & $\mathrm{P}$ & $21 \mathrm{pt} / 51 \mathrm{~d}$ & $\mathrm{E}$ & $\mathrm{Y}$ & $\mathrm{Y}$ & $\mathrm{Y}$ & $\mathrm{N}$ & $\mathrm{Y}$ \\
\hline Simmons et al (128) & $4 / 5$ & $11 / 14$ & $\mathrm{P}$ & 164 & $\mathrm{E}$ & $\mathrm{Y}$ & $\mathrm{U}$ & $\mathrm{N}$ & $\mathrm{Y}$ & $\mathrm{Y}$ \\
\hline Smith et al (129) & $4 / 5$ & $11 / 14$ & $\mathrm{R}$ & 72 & $\mathrm{E}$ & $\mathrm{Y}$ & $\mathrm{U}$ & $\mathrm{Y}$ & $\mathrm{Y}$ & $\mathrm{Y}$ \\
\hline Smith et al (130) & $3 / 5$ & $11 / 14$ & $\mathrm{R}$ & 36 & $\mathrm{E}$ & $\mathrm{Y}$ & $\mathrm{U}$ & $\mathrm{N}$ & $\mathrm{N}$ & $\mathrm{N}$ \\
\hline Vanharanta et al (131) & $4 / 5$ & $11 / 14$ & $\mathrm{P}$ & $291 / 790$ & $\mathrm{E}$ & $\mathrm{Y}$ & $\mathrm{Y}$ & $\mathrm{Y}$ & $\mathrm{Y}$ & $\mathrm{Y}$ \\
\hline Vanharanta et al (32) & $4 / 5$ & $11 / 14$ & $\mathrm{P}$ & $91 / 290$ & $\mathrm{E}$ & $\mathrm{Y}$ & $\mathrm{Y}$ & $\mathrm{Y}$ & $\mathrm{Y}$ & $\mathrm{Y}$ \\
\hline Vanharanta et al (132) & $4 / 5$ & $11 / 14$ & $\mathrm{P}$ & 107 & $\mathrm{E}$ & $\mathrm{Y}$ & $\mathrm{Y}$ & $\mathrm{Y}$ & $\mathrm{Y}$ & $\mathrm{Y}$ \\
\hline Vanaharanta et al (133) & $4 / 5$ & $11 / 14$ & $\mathrm{P}$ & $300 / 816$ & $\mathrm{E}$ & $\mathrm{Y}$ & $\mathrm{Y}$ & $\mathrm{Y}$ & $\mathrm{Y}$ & $\mathrm{Y}$ \\
\hline Weishaupt et al (134) & $4 / 5$ & $11 / 14$ & $\mathrm{P}$ & $50 / 122$ & $\mathrm{E}$ & $\mathrm{Y}$ & $\mathrm{N}$ & $\mathrm{N}$ & $\mathrm{Y}$ & $\mathrm{Y}$ \\
\hline Walsh et al (135) & $5 / 5$ & $13 / 14$ & $\mathrm{P}$ & $10 \mathrm{Asx} / 7 \mathrm{Sx}$ & $\mathrm{E}$ & $\mathrm{Y}$ & $\mathrm{Y}$ & $\mathrm{N}$ & $\mathrm{N}$ & $\mathrm{Y}$ \\
\hline Wetzel et al (136) & $4 / 5$ & $11 / 14$ & $\mathrm{R}$ & 48 & $\mathrm{E}$ & $\mathrm{Y}$ & $\mathrm{N}$ & $\mathrm{N}$ & $\mathrm{N}$ & $\mathrm{Y}$ \\
\hline Yrjama et al (138) & $3 / 5$ & $8 / 14$ & $\mathrm{P}$ & 38 & $\mathrm{U}$ & $\mathrm{Y}$ & $\mathrm{Y}$ & $\mathrm{N}$ & $\mathrm{Y}$ & $\mathrm{Y}$ \\
\hline Zucherman et al (140) & $3 / 5$ & $9 / 14$ & $\mathrm{R}$ & 18 & $\mathrm{U}$ & $\mathrm{Y}$ & $\mathrm{Y}$ & $\mathrm{Y}$ & $\mathrm{Y}$ & $\mathrm{N}$ \\
\hline
\end{tabular}

$R A=$ randomized $R=$ retrospective $P=$ prospective $C=$ controlled $; C C=$ case controlled $B=$ blinded $; Y=$ yes $;=$ no; $E=$ extrapedicular $I=$ interlaminar $L=$ left; $U=$ unknown; $U A O=$ side unknown, anterior oblique; $R A O=$ right anterior oblique; Asx = asymptomatic; $S X=$ symptomatic; \#d=number of discs 
at varying pressures. In this latter group, high pressures induced pain; even then, the pain was mild. The authors generated a receiver operator characteristic curve and a table displaying the probability of experiencing a specified pain intensity versus a specified pressure. From their data alone, the false positive rate could be reduced to less than $10 \%$. Particular scenarios included 1) $\leq 20$ psi above opening pressure and a pain numerical rating scale (NRS) $>0$; and 2) $\geq 0$ psi above opening pressure and NRS $\geq 4$. They subsequently combined their data with Carragee et al (89) and developed operational criteria for discography. For an individual disc, if the evoked pain-NRS $\geq 4$ at $\leq 50$ psi above opening pressure (aop), the false-positive rate can be reduced to $\leq 10 \%$; if the evoked pain-NRS $\geq 4$ at $\leq 30$ psi aop OR if the evoked pain-NRS $\geq 6$ AT $\leq 50$ psi aop, the false positive rate is reduced to $0 \%$.

Derby et al (76) performed pressure-controlled discography in 16 volunteers without low back pain (55 discs) and in 90 chronic low back pain patients (282 discs). The purpose of this prospective study was to determine if discography could distinguish asymptomatic from symptomatic discs, in those that are morphologically abnormal (Grade 3 annular disruption, only). A negative disc was defined as one in which pain was absent or "unfamiliar"; if the discogram, however, produced any severe pain, NRS $\geq 6$ at pressures $\leq 50$ psi above opening pressure and at volumes $\leq 3.5 \mathrm{ml}$, this disc was excluded from the negative group. In the volunteer group, $58.2 \%$ of discs had Grade III annular tears and all of these discs were negative. In the chronic low back pain group, $70.6 \%$ of discs had Grade III annular tears; in these Grade III discs, $52.3 \%$ were negative and $47.7 \%$ were positive. Please note that this "positive" group simply included those discs that did not meet the negative response criteria. Notably, a number of discs in this study's positive group would not meet the operational criteria for positivity established in two other papers published by Derby et al $(75,77)$. For instance, a patient with familiar pain at pressures $>50$ psi or NRS $<6$ would qualify as a 'positive' disc in this paper, unlike Derby et al (77). In summary, the authors found that discs with Grade III annular tears exhibit different pain provocation characteristics. Control and negative discs displayed similar traits in terms of evoked pain intensity. Evoked pain intensity for positive discs was significantly higher than control or negative discs. They also stated that pressure-controlled manometric discography using strict criteria may distinguish asymptomatic discs among morphologically abnormal discs with Grade 3 annular tears in patients with chronic discogenic LBP.

Derby et al (77) performed pressure-controlled discography on 86 chronic low back pain patients (279 discs). In a cross-sectional analysis of prospectively acquired data, they analyzed the relationship between computed tomography disc morphology - annular disruption and discography findings. Using strict operational criteria (75), a symptomatic disc was one with evoked pain-NRS $\geq 6$ at $\leq 50$ psi above opening pressure. They demonstrated a correlation between annular disruption grade and the rate of symptomatic discs. $44 \%$ of discs with an annular disruption grade $\geq 3$ are symptomatic. Negligible rates of symptomatic discs were encountered at annular disruption grades $\leq 2$. Furthermore, anatomically normal discs - annular disruption grade 0 -were never symptomatic. Severe pain intensity was proportional to pressure in Grade 4 (annular disruption and circumferential contrast extension) and Grade 5 (contrast leakage through the outer annulus) discs. The authors concluded that an annular disruption extending to, and beyond, the outer annulus is a key factor in pain generation. The authors suggest that the specificity of discography can be improved, if a positive disc is defined as one in which, 1) evoked pain-NRS $\geq 6$ at $\leq 50$ psi above opening pressure; and 2) the morphology is abnormal - high grade annular disruption.

Lim et al (78), in a prospective study, studied 47 patients who underwent discography followed by $\mathrm{CT}$. They then looked at the prior MRI and post discogram CT images of patients who had positive or concordant painful discs. Typical MRI findings in patients with concordant pain at discography include grade 4 or 5 disc degeneration and presence of a high intensity zone. Typical CT discography findings with concordant pain were fissured/ruptured discs and contrast extending into/beyond the outer annulus on CT.

Peng et al (79) conducted a prospective study of lumbar discography on 52 patients, but reported the results of a subset of 17 patients with single level high intensity zones (HIZ) on MRI. Based on the findings of discography, post-discography CT scanning, and postfusion, histopathological analysis of the intervertebral disc, the authors concluded that HIZ zones reproduce concordant pain during discography. Furthermore, the HIZ corresponds to a strip of vascularized, granulation tissue in the outer annulus.

Shin et al (80) conducted a prospective study of 
lumbar discography on 21 patients and 51 discs. A positive disc was one in which, 1) pain was concordant or similar; 2) pain intensity $\geq 6 / 10 ; 3$ ) pressure above opening pressure $\leq 50 \mathrm{psi}$; and 4 ) total volume injected $\leq 3$ $\mathrm{ml}$. All patients underwent $\mathrm{CT}$ scanning, following discography. The authors noted a progressive reduction in elastance ( $\Delta$ pressure/ $\Delta$ volume), with increasing grades of annular degeneration. There was a significant difference between elastance of Grade 0 (36.8) and Grade 5 (17.0) discs. In other words, the annular disruption grade is proportional to compliance. All positive discs demonstrated Grade 3 or higher annular disruptions. All Grade 0 discs were negative. Pain responses correlated with intradiscal pressure. Grade 3 and Grade 5 discs evoked pain at lower pressures in positive discs, relative to negative discs. In fact, positive discs evoked a higher intensity of pain, as compared to negative discs. The authors concluded that discography was a useful tool to diagnose discogenic pain and to guide surgical therapy in conjuction with CT morphology disc.

\section{Strength of Evidence}

There is strong evidence for the utility of discography as an IVD imaging tool. There is strong evidence that intradiscal distention can produce pain. There is strong evidence supporting the role of discography in identifying patients with chronic lumbar discogenic pain. There is moderate evidence supporting the role of discography in identifying patients with chronic cervical discogenic pain. There is limited evidence supporting the role of discography in identifying patients with chronic thoracic discogenic pain.

\section{Discussion}

Deeks (141) provided a framework to evaluate a diagnostic study's quality and likelihood of bias. Unlike the AHRQ (67) or the QUADAS (68), this involved looking at only 3 criteria: 1) patient sample, 2) reference standard, and 3) index test. Deeks (141) recommended optimal patient recruitment, optimal execution of the index test, and optimal execution of the reference standard. The majority of the papers met 2 of these criteria, but only a few met 3 . All papers met at least $50 \%$ of the AHRQ or QUADAS criteria $(67,68)$.

Knottnerus et al (142) stated that there are several methodological challenges that must be addressed in diagnostic accuracy studies. These include the gold standard problem, spectrum and selection bias, "soft" measures (subjective phenomena), observer variability and bias, complex relations, clinical impact, sample size, and the rapid progress of knowledge (142). In this systematic review, we address these concerns, but unfortunately, most discography studies cannot overcome these methodological limitations.

There is no gold standard for discogenic pain and thus, we considered pathological disc morphology to be the gold standard. Treatment, particularly a controversial treatment, should not serve as the gold standard for a diagnostic test. Diagnostic tests detect the presence or absence of a disease process/ entity. The presence or absence of treatment should not influence whether or not the disease entity exists. Some authors, implicitly, assume that discography is a pre-surgical screening tool. This suggests that the validity of discography depends on the outcome of a controversial treatment: spinal fusion (74). If this concept is generalized, one could challenge the validity of any diagnostic test by the treatment outcome. To use a controversial treatment such as lumbar spinal fusion for discogenic pain to evaluate the worthiness of discography as a test for discogenic pain seems flawed. The treatment of discogenic pain continues to be a frustrating endeavor and no treatment has been found yet that provides significant relief to a majority of presumed discogenic back pain patients on a consistent basis. In our opinion, abnormal disc morphology should still serve as the gold standard and not the response to a presumed treatment for a disease that is particularly difficult to treat.

The majority of the selected studies were able to describe, in detail, the index test (assessment of pain) and reference or gold standard test (assessment of disc morphology). All the studies suffered from selection bias and a few from spectrum bias. The assessment of pain is a soft measure and a few papers challenged the ability of a patient to report pain, consistently, during discography. Observer variability and observer bias were present in all papers; only a few attempted to minimize these factors. These few studies attempted blinding in which independent observers reviewed either the patient's videotape or the discographic images. Inter-observer and intra-observer validation of discographic imaging was performed in only a few studies. Inter-observer validation of pain response was performed in only a few studies; since pain is a subjective experience, the utility of this latter validation scheme is questionable. Complex relations were not accounted for in all studies, since discography is no longer performed as an isolated test. Discography is performed on patients with persistent 
spinal pain, after a number of other tests have been ordered. Sample sizes for most studies were small. In terms of patient selection, methodology was poor for most papers and all papers could be criticized for selection bias. Nonetheless, ethical barriers prevent performing invasive tests on large patient samples that may or may not have disease. This is why most discography studies were retrospective or prospective, but not randomized and double blinded. A number of studies presented data in $2 \times 2$ contingency table format. Since most discography results are presented as positive or negative, a $2 \times 2$ format is useful to readers; this format allows comparison between different studies. Finally, the rapid progress in our understanding of pain and pain processing may impact the diagnostic accuracy of discography.

Overall, discography is a useful imaging tool. Intradiscal pathology may be missed by other studies. A post-discography CT scan may provide useful, additional information, particularly to differentiate annular tears from annular disruption. Discography can produce pain in patients with mild or chronic low back pain, with a chronic pain disorder, or with no pain at all. Discography rarely provokes pain in morphologically normal discs. Annular tears, particularly those extending to the outer annulus, are often associated with discographically provoked pain. Degenerative disc changes are not necessarily associated with pain provocation. However, progressive degeneration is associated with dissimilar and similar pain provocation.

Discography cannot predict future back pain problems or disability in asymptomatic patients. Discography should not be used as a screening test for employment. Patients with persistent back pain may have emotional and psychological problems which can influence future outcomes such as response to therapy and the development of disability due to chronic back pain. False-positives may be reduced during discography if only patients with normal psychometric profiles and without other chronic pain syndromes or prior lumbar spine surgery are selected. The background incidence of minor back pain that is discogenically mediated may be high and may confound the identification of those patients with severe discogenic pain.

If one considers discography to be a form of pressure pain threshold (PPT), then one would expect both normal and abnormal discs to produce pain. All tissues can evoke pain if exposed to sufficiently high pressures. In this scenario, one cannot arbitrarily decide between a true-positive versus a false-positive, in an a priori fashion; in a symptomatic low back pain population, we cannot define false-positive rates based on pain provocation in an asymptomatic population. Rather, to define normality, one would have to tabulate PPTs in a large population-based fashion. One example, as suggested by O'Neill and Kurgansky (113) would be to plot the number of individuals ( $y$-axis) versus PPTs ( $x$-axis). This method could be applied prospectively to an asymptomatic population with normal psychological profiles, an asymptomatic population with abnormal psychological profiles, an asymptomatic population with chronic non-spinal pain, and a symptomatic spinal pain population. Based on these population distributions, one could then define the probability of a true- or false-positive in an individual patient. Similar strategies are used in screening mothers during their first trimester, the triple screen, to determine their risk of delivering a baby with Down's syndrome $(143,144)$.

We can extend this concept further. We could define discography results as numerical values rather than as a binary, positive or negative, result. In other words, if discography results were reported in terms of PPTs, the sensitivity and specificity would change at different PPT cutoffs. Then, the sensitivity (y-axis) could be plotted versus 1 -specificity ( $x$-axis) by using the results obtained at different PPT cutoffs. This will define a receiver operator characteristic (ROC) curve, which is an effective method for evaluating the quality of a diagnostic test (145-147). If the ROC curve passes upwards to the left, the diagnostic test is nearly perfect in distinguishing disease from no disease. If the ROC curve is diagonal ( 45 degrees), the diagnostic test's ability to detect disease will equal that of an educated guess -- a guess based on prevalence data $(143,145-147)$. ROC curves can be generated by using prospectively collected pressure pain threshold data from discography. Our 2005 systematic review recommended ROC curves for discography (1). Only one paper, thus far, has done this. Derby et al (77) used this ROC curve to develop strict operational criteria for defining a symptomatic disc. They correlated this information with abnormal disc morphology (75). When paired together, the false positive rate could be significantly reduced during the performance of discography. Future discography studies should consider this model and generate ROC curves, in order to validate the findings by Derby et al (75-77).

In the article by Carragee et al (73) evaluating low-pressure discography in patients without 
significant low back pain, it is worth noting that this is a retrospective study evaluating data used in 3 prior studies. From these data, 69 volunteers in 4 study groups were defined. Only the 10 patients in the first group were truly asymptomatic, without LBP or any chronic pain condition. A second group of 14 patients did not have LBP but did have some other chronic pain condition. More significantly, the third group consisted of 20 patients who had undergone lumbar laminectomy previously but were now pain free. The last group included 25 patients with ongoing clinically insignificant backache. Thus, 45 of the 69 patients, or $65 \%$ of patients, had a preexisting condition of the lumbar spine, whether due to prior surgery or backache. In contrast, in Derby's two articles that included asymptomatic subjects $(75,76)$, the asymptomatic individuals were without present LBP problems and were allowed no more than 3 episodes of LBP in the previous year in 1 study (75) and no more than 2 episodes of LBP in the previous year in the second (76). Of interest is the fact that none of the patients in the Carragee's subgroup of truly asymptomatic patients (Group One) had a lowpressure positive discogram. The group with the largest number of low-pressure positive discograms was the second group with 5 of 14 subjects positive, or $36 \%$. The overall number of low-pressure positive subjects was 17 of 69 patients or $25 \%$. One can see that the whole study group was not entirely asymptomatic. The authors state that their findings continue to offer support for the conclusion that subjects with neither LBP, a chronic pain state, nor previous surgery have a low risk for low-pressure painful injections. Despite their conclusions, the authors' data suggest that truly asymptomatic patients (and discs) are at a negligible risk for discography-induced pain, at low pressures. Nonetheless, they estimate the risk of false-positives in this group at approximately $25 \%$. This is much higher than the $10 \%$ or less false-positive rate that Derby suggests is attainable when strict pressure and pain score criteria are used (75). These criteria are listed above in the Results section (75).

In the absence of population-based data, the ability of discography to provoke pain should not be surprising. The more difficult questions are: 1) whether discography can select patients for treatment?; 2) whether discography can predict outcomes following surgical and non-surgical treatments?; and 3) whether symptomatic internal disc disruption exists, as a discrete anatomic/structural entity, in isolation from peripheral and central pain processes?

Future research is needed that investigates the precise mechanisms of how discography induces pain and how this correlates with functional activities. Also needed is external validation, not based on subjective pain assessments, of the ability of discography to precisely identify the disc as the pain generator. In those patients with chronic spinal pain, perhaps advances in functional imaging, pain processing neurobiology, and clinical biomarkers can complement discography, in ascertaining the relative contribution of the IVD to pain. Lastly, population-based data on intradiscal pressure pain thresholds, both in asymptomatic and symptomatic patients, would enable practitioners to distinguish between true- and false-positives in individual patients. Ideally, such pressure pain thresholds could be used to generate a receiver operator characteristic curve to better evaluate the diagnostic accuracy of discography.

In the interim, questioning the validity of discography warrants questioning the role of the intervertebral disc as a discrete pain generator, or more specifically, challenges the concept of symptomatic internal disc disruption. If one considers discography to be a useless test, then one may have to abandon the concept of the IVD as a discrete pain generator and abandon the pursuit of intradiscal therapies, whether surgical or non-surgical.

\section{Conclusion}

There is strong evidence for the utility of discography as an IVD imaging tool. There is strong evidence that intradiscal distention can produce pain. There is strong evidence supporting the role of discography in identifying patients with chronic lumbar discogenic pain. There is moderate evidence supporting the role of discography in identifying patients with chronic cervical discogenic pain. There is limited evidence supporting the role of discography in identifying patients with chronic thoracic discogenic pain. The recent generation of a receiver operator characteristic curve for discography should serve as a model for future discography studies and may provide a practical tool for discographers. 


\section{References}

1. Shah RV, Everett C, McKenzie-Brown A, Sehgal N. Discography as a diagnostic test for spinal pain: A systematic and narrative review. Pain Physician 2005; 8:187-209.

2. Boswell MV, Shah RV, Everett CR, Sehgal N, Mckenzie-Brown AM, Abdi S, Bowman RC, Deer TR, Datta S, Colson JD, Spillane WF, Smith HS, LucasLevin LF, Burton AW, Chopra P, Staats PS, Wasserman RA, Manchikanti L. Interventional techniques in the management of chronic spinal pain: Evidencebased practice guidelines. Pain Physician 2005; 8:1-47.

3. Cohen SP, Larkin TM, Barna SA, Palmer WE, Hecht AC, Stojanovic MP. Lumbar discography: A comprehensive review of outcome studies, diagnostic accuracy, and principles. Reg Anesth Pain Med 2005; 30:163-183.

4. Bogduk N, Modic MT. Lumbar discography. Spine 1996; 21:402-404.

5. Guyer RD, Ohnmeiss DD; NASS. Lumbar discography. Spine J 2003; 3:11S$27 \mathrm{~S}$.

6. Guyer RD, Ohnmeiss DD. Lumbar discography. Position statement from the North American Spine Society Diagnostic and Therapeutic Committee. Spine 1995; 20:2048-2059.

7. Smith GW, Nichols P Jr. The technique of cervical discography. Radiology 1957; 68:718-720.

8. Cloward RB. The anterior surgical approach to the cervical spine: the Cloward Procedure: past, present, and future. The presidential guest lecture, Cervical Spine Research Society. Spine 1988; 13:823-827.

9. Cloward RB. Anterior herniation of a ruptured lumbar intervertebral disk; comments on the diagnostic value of the diskogram. AMA Arch Surg 1952; 64:457-463.

10. Lindblom K. Technique and results of diagnostic disc puncture and injection (discography) in the lumbar region. Acta Orthop Scand 1951; 20:315-326.

11. Lindblom K. Technique and results in myelography and disc puncture. Acta Radiol 1950; 34:321-330.

12. Antti-Poika I, Soini J, Tallroth K, Yrjonen T, Konttinen YT. Clinical relevance of discography combined with CT scanning. A study of 100 patients. J Bone Joint Surg Br 1990; 72:480-485.

13. Buirski G, Silberstein $M$. The symptomatic lumbar disc in patients with lowback pain. Magnetic resonance imaging appearances in both a symptomat- ic and control population. Spine 1993; 18:1808-1811.

14. Brightbill TC, Pile N, Eichelberger RP, Whitman M Jr. Normal magnetic resonance imaging and abnormal discog. raphy in lumbar disc disruption. Spine 1994; 19:1075-1077.

15. Birney TJ, White JJ Jr, Berens D, Kuhn G. Comparison of MRI and discography in the diagnosis of lumbar degenerative disc disease. I Spinal Disord 1992; 5:417-423.

16. Gibson MJ, Buckley J, Mawhinney R, Mulholland RC, Worthington BS. Magnetic resonance imaging and discography in the diagnosis of disc degeneration. A comparative study of 50 discs. J Bone Joint Surg Br 1986; 68:369-373.

17. Yu SW, Haughton VM, Sether LA, Wagner M. Comparison of MR and diskography in detecting radial tears of the annulus: A postmortem study. AJNR Am J Neuroradiol 1989; 10:1077-1081.

18. Abdelwahab IF, Gould ES. The role of diskography after negative postmyelography CT scans: Retrospective review. AJNR Am J Neuroradiol 1988; 9:187-190.

19. Videman T, Nurminen $M$. The occurrence of annular tears and their relation to lifetime back pain history: a cadaveric study using barium sulfate discography. Spine 2004; 29:2668-2676.

20. Adams MA, Dolan P, Hutton WC. The stages of disc degeneration as revealed by discograms. J Bone Joint Surg Br 1986; 68:36-41.

21. Milette PC, Fontaine S, Lepanto L, Cardinal E, Breton G. Differentiating lumbar disc protrusions, disc bulges, and discs with normal contour but abnormal signal intensity. Magnetic resonance imaging with discographic correlations. Spine 1999; 24:44-53.

22. Bernard TN Jr. Lumbar discography followed by computed tomography. Refining the diagnosis of low-back pain. Spine 1990; 15:690-707.

23. Sachs BL, Vanharanta H, Spivey MA, Guyer RD, Videman T, Rashbaum RF, Johnson RG, Hochschuler SH, Mooney V. Dallas discogram description. A new classification of CT/discography in lowback disorders. Spine 1987; 12:287294.

24. McCutcheon ME, Thompson WC III. CT scanning of lumbar discography. A useful diagnostic adjunct. Spine 1986; 11:257-259.

25. Block AR, Vanharanta H, Ohnmeiss DD, Guyer RD. Discographic pain report. In- fluence of psychological factors. Spine 1996; 21:334-338.

26. Adams MA, Roughley PJ. What is intervertebral disc degeneration, and what causes it? Spine 2006; 31:2151-2161.

27. Adams MA, Freeman BJ, Morrison HP, Nelson IW, Dolan P. Mechanical initiation of intervertebral disc degeneration. Spine 2000; 25:1625-1636.

28. Battie MC, Videman T, Gibbons LE, Fisher LD, Manninen H, Gill K. 1995 Volvo Award in clinical sciences. Determinants of lumbar disc degeneration. A study relating lifetime exposures and magnetic resonance imaging findings in identical twins. Spine 1995; 20:26012612.

29. Battie MC, Videman T, Parent E. Lumbar disc degeneration: epidemiology and genetic influences. Spine 2004; 29:2679-2690.

30. Rajasekaran S, Babu JN, Arun R, Armstrong BR, Shetty AP, Murugan S. ISSLS prize winner: A study of diffusion in human lumbar discs: a serial magnetic resonance imaging study documenting the influence of the endplate on diffusion in normal and degenerate discs. Spine 2004; 29:2654-2667.

31. Moneta GB, Videman T, Kaivanto K, Aprill C, Spivey M, Vanharanta H, Sachs BL, Guyer RD, Hochschuler SH, Raschbaum RF, Mooney V. Reported pain during lumbar discography as a function of annular ruptures and disc degeneration. A re-analysis of 833 discograms. Spine 1994; 19:1968-1974.

32. Vanharanta H, Sachs BL, Spivey MA, Guyer RD, Hochschuler SH, Rashbaum RF, Johnson RG, Ohnmeiss D, Mooney V. The relationship of pain provocation to lumbar disc deterioration as seen by CT/discography. Spine 1987; 12:295-298.

33. Slipman CW, Patel RK, Zhang L, Vresilovic E, Lenrow D, Shin C, Herzog R. Side of symptomatic annular tear and site of low back pain: Is there a correlation? Spine 2001; 26:E165-E169.

34. Aoki Y, Ohtori S, Takahashi K, Ino H, Takahashi Y, Chiba T, Moriya H. Innervation of the lumbar intervertebral disc by nerve growth factor-dependent neurons related to inflammatory pain. Spine 2004; 29:1077-1081.

35. Aoki Y, Takahashi Y, Takahashi K, Chiba T, Kurokawa M, Ozawa T, Moriya $\mathrm{H}$. Sensory innervation of the lateral portion of the lumbar intervertebral disc in rats. Spine J 2004; 4:275-280.

36. Aoki Y, Takahashi Y, Ohtori S, Moriya H, Takahashi K. Distribution and immuno- 
cytochemical characterization of dorsal root ganglion neurons innervating the lumbar intervertebral disc in rats: A review. Life Sci 2004; 74:2627-2642.

37. Takahashi Y, Chiba T, Kurokawa M, Aoki Y, Takahashi K, Yamagata M. Stereoscopic structure of sensory nerve fibers in the lumbar spine and related tissues. Spine 2003; 28:871-880.

38. Nakamura SI, Takahashi K, Takahashi Y, Yamagata M, Moriya $H$. The afferent pathways of discogenic low-back pain. Evaluation of L2 spinal nerve infiltration. J Bone Joint Surg Br 1996; 78:606612.

39. Nakamura S, Takahashi K, Takahashi Y, Morinaga T, Shimada Y, Moriya H. Origin of nerves supplying the posterior portion of lumbar intervertebral discs in rats. Spine 1996; 21:917-924.

40. Mendez R, Bailey S, Paine G, Mazzilli M, Stedje-Larsen E, Nance B, Dietrick K. Evaluation of the L2 spinal nerve root infiltration as a diagnostic tool for discogenic low back pain. Pain Physician 2005; 8:55-59.

41. Simopoulous TT, Malik AB, Sial KA, Elkersh M, Bajwa ZH. Radiofrequency lesioning of the $\mathrm{l} 2$ ramus communicans in managing discogenic low back pain. Pain Physician 2005; 8:61-65.

42. Suseki K, Takahashi Y, Takahashi K, Chiba T, Yamagata M, Moriya H. Sensory nerve fibers from lumbar intervertebral discs pass through rami communicantes. A possible pathway for discogenic low back pain. J Bone Joint Surg $\mathrm{Br}$ 1998; 80:737-742.

43. Ohtori S, Takahashi Y, Takahashi K, Yamagata M, Chiba T, Tanaka K, Hirayama J, Moriya H. Sensory innervation of the dorsal portion of the lumbar intervertebral disc in rats. Spine 1999; 24:2295-2299.

44. Freemont AJ, Peacock TE, Goupille P, Hoyland JA, O’Brien J, Jayson MI. Nerve ingrowth into disease intervertebral disc in chronic back pain. Lancet 1997; 350:178-181.

45. Kuslich SD, Ulstrom CL, Michael CJ. The tissue origin of low back pain and sciatica: A report of pain response to tissue stimulation during operation on the lumbar spine using local anesthesia. Orthop Clin North Am 1991; 22:181187.

46. Groen GJ, Baljet B, Drukker J. Nerves and nerve plexuses of the human vertebral column. Am J Anat 1990; 188:286296.

47. Ozawa T, Ohtori S, Inoue G, Aoki Y, Moriya H, Takahashi K. The degenerat- ed lumbar intervertebral disc is innervated primarily by peptide-containing sensory nerve fibers in humans. Spine 2006; 31:2418-2422.

48. Aoki Y, Akeda K, An H, Muehleman C, Takahashi K, Moriya H, Masuda K. Nerve fiber ingrowth into scar tissue formed following nucleus pulposus extrusion in the rabbit anular-puncture disc degeneration model: Effects of depth of puncture. Spine 2006; 31: E774-E780.

49. Bogduk N, Tynan W, Wilson AS. The nerve supply to the human lumbar intervertebral discs. J Anat 1981; 132:39-56.

50. Averill S, McMahon SB, Clary DO, Reichardt LF, Priestley JV. Immunocytochemical localization of trkA receptors in chemically identified subgroups of adult rat sensory neurons. Eur J Neurosci 1995; 7:1484-1494.

51. Ohtori S, Takahashi K, Chiba T, Yamagata M, Sameda H, Moriya H. Substance $\mathrm{P}$ and calcitonin gene-related peptide immunoreactive sensory DRG neurons innervating the lumbar intervertebral discs in rats. Ann Anat 2002; 184:235240.

52. Ashton IK, Roberts S, Jaffray DC, Polak $J M$, Eisenstein SM. Neuropeptides in the human intervertebral disc. J Orthop Res 1994; 12:186-192.

53. Urasaki T, Muro T, Ito S, Hattori Y, Ozaki S. Consistency of lumbar discograms of the same disc obtained twice at a 2week interval: Influence of needle tip position. J Orthop Sci 1998; 3:243-251.

54. Bogduk N. Lumbar disc stimulation (provocation discography). In Practice Guidelines for Spinal Diagnostic and Treatment Procedures, 1st edition. International Spine Intervention Society, 2004, pp 20-46.

55. Bogduk N. Thoracic provocation discography. In Practice Guidelines for Spinal Diagnostic and Treatment Procedures, 1st edition. International Spine Intervention Society, 2004, pp 287-294.

56. Bogduk N. Cervical disc stimulation (provocation discography). In Practice Guidelines for Spinal Diagnostic and Treatment Procedures, 1st edition. International Spine Intervention Society, 2004, pp 95-111.

57. Merskey H, Bogduk N. Classification of Chronic Pain. Descriptions of Chronic Pain Syndromes and Definition of Pain Terms, 2nd ed. IASP Press, Seattle, 1994.

58. Appleby D, Andersson G, Totta M. Metaanalysis of the efficacy and safety of intradical electrothermal therapy (IDET).
Pain Med 2006; 4:308-316.

59. Thalgott JS, Albert TJ, Vaccaro AR, Aprill CN, Giuffre JM, Drake JS, Henke JP. A new classification system for degenerative disc disease of the lumbar spine based on magnetic resonance imaging, provocative discography, plain radiographs and anatomic considerations. Spine J 2004; 4:167S-172S.

6o. Shuff C, An HS. Artificial disc replacement: the new solution for discogenic low back pain? Am J Orthop 2005; 34:812.

61. Pauza KJ, Howell S, Dreyfuss P, Peloza JH, Dawson K, Bogduk N. A randomized, placebo-controlled trial of intradiscal electrothermal therapy for the treatment of discogenic low back pain. Spine / 2004; 4:27-35.

62. Saal JA, Saal JS. Intradiscal electrothermal treatment for chronic discogenic low back pain: prospective outcome study with a minimum 2-year followup. Spine 2002; 27:966-973.

63. Andersson GB, Mekhail NA, Block JE. Treatment of intractable discogenic low back pain. A systematic review of spinal fusion and intradiscal electrothermal therapy (IDET). Pain Physician 2006; 9:237-248.

64. Huang TS, Zucherman JF, Hsu KY, Shapiro M, Lentz D, Gartland J. Gadopentetate dimeglumine as an intradiscal contrast agent. Spine 2002; 27:839-843.

65. Simmons JW, Aprill CN, Dwyer AP, Brodsky AE. A reassessment of Holt's data on: "The question of lumbar discography." Clin Orthop Relat Res 1988; 237:120-124.

66. Grubb SA, Kelly CK. Cervical discography: clinical implications from 12 years of experience. Spine 2000; 25:13821389.

67. West S, King V, Carey TS, Lohr KN, McKoy,N, Sutton SF, Lux L. Systems to rate the strength of scientific evidence. Evidence Report/Technology Assessment No. 47 University of North Carolina: Agency for Healthcare Research and Quality. AHRQ Publication No. 02E016; April 2002.

68. Whiting P, Rutjes AW, Reitsma JB, Bossuyt PM, Kleijnen J. The development of QUADAS: a tool for the quality assessment of studies of diagnostic accuracy included in systematic reviews. BMC Med Res Methodol 2003; 3:25.

69. Manchikanti L, Abdi S, Lucas L. Evidence synthesis and development of guidelines in interventional pain management: An invited review. Pain Physician $2005 ; 8: 73-86$. 
70. Gill K, Blumenthal SL. Functional results after anterior lumbar fusion at $\mathrm{L}_{5}-\mathrm{S}_{1}$ in patients with normal and abnormal MRI scans. Spine 1992; 17:940942.

71. Blankenbaker DG, Haughton VM, Rogers BP, Meyerand ME, Fine JP. Axial rotation of the lumbar spinal motion segments correlated with concordant pain on discography. A preliminary study. AJR 2006; 186:795-799.

72. Carragee EJ, Alamin TF, Miller JL, Carragee JM. Discographic, MRI, and psychosocial determinants of low back pain disability and remission: a prospective study in subjects with benign persistent back pain. Spine J 2005; 5:24-35.

73. Carragee EJ, Alamin TF, Carragee JM. Low-pressure positive discography in subjects asymptomatic of significant low back pain illness. Spine 2006; 31:505-509.

74. Carragee EJ, Lincoln T, Parmar VS, Alamin T. A gold standard evaluation of the "discogenic pain" diagnosis as determined by provocative discography. Spine 2006; 31:2115-2123.

75. Derby R, Lee SH, Kim BJ, Chen Y, Aprill C, Bogduk N. Pressure-controlled lumbar discography in volunteers with low back pain symptoms. Pain Med 2005; 6:213-221.

76. Derby R, Kim BJ, Lee SH, Chen Y, Seo KS, Aprill C. Comparison of discographic findings in asymptomatic subject discs and negative discs of chronic LBP patients: Can discography distinguish asymptomatic discs among morphologically abnormal discs? Spine J 2005; 5:389-394.

77. Derby R, Kim BJ, Chen Y, Seo KS, Lee $\mathrm{SH}$. The relation between annular disruption on computed tomography scan and pressure-controlled diskography. Arch Phys Med Rehabil 2005; 86:15341538.

78. Lim CH, Jee WH, Son BC, Kim DH, Ha KY, Park CK. Discogenic lumbar pain: association with MR imaging and $\mathrm{CT}$ discography. Eur J Radiol 2005; 54:431-437.

79. Peng B, Hou S, Wu W, Zhang C, Yang Y. The pathogenesis and clinical significance of a high-intensity zone ( $\mathrm{HIZ}$ ) of lumbar intervertebral disc on MR imaging in the patient with discogenic low back pain. Eur Spine J 2006; 15:583587.

80. Shin DA, Kim HI, Jung JH, Shin DG, Lee JO. Diagnostic relevance of pressurecontrolled discography. J Korean Med Sci 2006; 21:911-916.

81. Aprill C, Bogduk N. High-intensity zone:
A diagnostic sign of painful lumbar disc on magnetic resonance imaging. $\mathrm{Br} J$ Radiol 1992; 65:361-369.

82. Bogduk N, Aprill C. On the nature of neck pain, discography and cervical zygapophysial joint blocks. Pain 1993; 54:213-217.

83. Braithwaite I, White J, Saifuddin A, Renton P, Taylor BA. Vertebral endplate (Modic) changes on lumbar spine MRI: Correlation with pain reproduction at lumbar discography. Eur Spine J 1998; 7:363-368.

84. Carragee EJ, Chen Y, Tanner CM, Hayward C, Rossi M, Hagle C. Can discography cause long-term back symptoms in previously asymptomatic subjects? Spine 2000; 25:1803-1808

85. Carragee EJ, Barcohana B, Alamin T, van den Haak E. Prospective controlled study of the development of lower back pain in previously asymptomatic subjects undergoing experimental discog. raphy. Spine 2004; 29:1112-1117.

86. Carragee EJ, Alamin TF, Miller J, Grafe M. Provocative discography in volunteer subjects with mild persistent low back pain. Spine J 2002; 2:25-34.

87. Carragee EJ, Chen Y, Tanner CM, Truong T, Lau E, Brito JL. Provocative discog. raphy in patients after limited lumbar discectomy: A controlled, randomized study of pain response in symptomatic and asymptomatic subjects. Spine 2000; 25:3065-3071.

88. Carragee EJ, Tanner CM, Yang B, Brito JL, Truong T. False-positive findings on lumbar discography. Reliability of subjective concordance assessment during provocative disc injection. Spine 1999; 24:2542-2547.

89. Carragee EJ, Tanner CM, Khurana S, Hayward C, Welsh J, Date E, Truong T, Rossi M, Hagle C. The rates of falsepositive lumbar discography in select patients without low back symptoms. Spine 2000; 25:1373-1380.

90. Carragee EJ, Paragioudakis SJ, Khurana S. 2000 Volvo Award winner in clinical studies: Lumbar high-intensity zone and discography in subjects without low back problems. Spine 2000; 25:2987-2992.

91. Cohen SP, Larkin T, Fant GV, Oberfoell $R$, Stojanovic $M$. Does needle insertion site affect diskography results? A retrospective analysis. Spine 2002; 27:2279-2283.

92. Colhoun E, McCall IW, Williams L, Cassar Pullicino VN. Provocation discography as a guide to planning operations on the spine. J Bone Joint Surg Br 1988;
70:267-271

93. Collins CD, Stack JP, O'Connell DJ, Walsh M, McManus FP, Redmond OM, Ennis JT. The role of discography in lumbar disc disease: a comparative study of magnetic resonance imaging and discography. Clin Radiol 1990; 42:252-257.

94. Connor PM, Darden BV II. Cervical discography complications and clinical efficacy. Spine 1993; 18:2035-2038.

95. Derby R, Howard MW, Grant JM, Lettice JJ, Van Peteghem PK, Ryan DP. The ability of pressure-controlled discography to predict surgical and nonsurgical outcomes. Spine 1999; 24:364-371.

96. Donelson R, Aprill C, Medcalf R, Grant W. A prospective study of centralization of lumbar and referred pain. A predictor of symptomatic discs and anular competence. Spine 1997; 22:11151122.

97. Greenspan A, Amparo EG, Gorczyca DP, Montesano PX. Is there a role for diskography in the era of magnetic resonance imaging? Prospective correlation and quantitative analysis of computed tomography-diskography, magnetic resonance imaging, and surgical findings. J Spinal Disord 1992; 5:26-31.

98. Heggeness MH, Watters WC III, Gray PM Jr. Discography of lumbar discs after surgical treatment for disc herniation. Spine 1997; 22:1606-1609.

99. Horton WC, Daftari TK. Which disc as visualized by magnetic resonance imaging is actually a source of pain? A correlation between magnetic resonance imaging and discography. Spine 1992; 17:S164-S171.

100. Ito $M$, Incorvaia KM, Yu SF, Fredrickson BE, Yuan HA, Rosenbaum AE. Predictive signs of discogenic lumbar pain on magnetic resonance imaging with discography correlation. Spine 1998; 23:1252-1258.

101. Lam KS, Carlin D, Mulholland RC. Lumbar disc high-intensity zone: the value and significance of provocative discography in the determination of the discogenic pain source. Eur Spine J 2000; 9:36-41.

102. Madan S, Gundanna M, Harley JM, Boeree NR, Sampson M. Does provocative discography screening of discogenic back pain improve surgical outcome? J Spinal Disord Tech 2002; 15:245-251.

103. Maezawa S, Muro T. Pain provocation at lumbar discography as analyzed by computed tomography/discography. Spine 1992; 17:1309-1315.

104. Manchikanti L, Singh V, Pampati VS, Fellows B, Beyer C, Damron K, Cash KA. 
Provocative discography in low back pain patients with or without somatization disorder: A randomized prospective evaluation. Pain Physician 2001; 4:227-239.

105. Manchikanti L, Singh V, Pampati VS, Damron KS, Barnhill RC, Beyer C, Cash KA. Evaluation of the relative contributions of various structures in chronic low back pain. Pain Physician 2001; 4:308-316.

106. McFadden JW. The stress lumbar discogram. Spine 1988; 13:931-933.

107. Milette PC, Fontaine S, Lepanto L, Breton G. Radiating pain to the lower extremities caused by lumbar disk rupture without spinal nerve root involvement. AJNR Am / Neuroradiol 1995 16:1605-1613.

108. Motimaya A, Arici M, George D, Ramsby $G$. Diagnostic value of cervical discography in the management of cervical discogenic pain. Conn Med 2000; 64:395-398.

109. Ohnmeiss DD, Guyer RD, Mason SL. The relation between cervical discographic pain responses and radiographic images. Clin J Pain 2000; 16:1-5.

110. Ohnmeiss DD, Vanharanta H, Guyer RD. The association between pain drawings and computed tomographic/discographic pain responses. Spine 1995; 20:729-733.

111. Ohnmeiss DD, Vanharanta $\mathrm{H}$, Ekholm J. Relation between pain location and disc pathology: a study of pain drawings and CT/discography. Clin J Pain 1999; 15:210-217.

112. Ohnmeiss DD, Vanharanta H, Ekholm J. Degree of disc disruption and lower extremity pain. Spine 1997; 22:1600-1605.

113. O'Neill C, Kurgansky M. Subgroups of positive discs on discography. Spine 2004; 29:2134-2139.

114. Osti OL, Fraser RD. MRI and discography of annular tears and intervertebral disc degeneration. A prospective clinical comparison. J Bone Joint Surg $\mathrm{Br}$ 1992; 74:431-435.

115. Parfenchuck TA, Janssen ME. A correlation of cervical magnetic resonance imaging and discography/computed tomographic discograms. Spine 1994; 19:2819-2825.

116. Parker LM, Murrell SE, Boden SD, Horton WC. The outcome of posterolateral fusion in highly selected patients with discogenic low back pain. Spine 1996; 21:1909-1916.

117. Ricketson R, Simmons JW, Hauser BO. The prolapsed intervertebral disc. The high-intensity zone with discography correlation. Spine 1996; 21:27582762.

118. Saifuddin A, Emanuel R, White J, Renton P, Braithwaite I, Taylor BA. An analysis of radiating pain at lumbar discog. raphy. Eur Spine J 1998; 7:358-362.

119. Saifuddin A, Braithwaite I, White J, Taylor BA, Renton P. The value of lumbar spine magnetic resonance imaging in the demonstration of anular tears. Spine 1998; 23:453-457.

120. Schechter NA, France MP, Lee CK. Painful internal disc derangements of the lumbosacral spine: discographic diagnosis and treatment by posterior lumbar interbody fusion. Orthopedics 1991; 14:447-451.

121. Schellhas KP, Pollei SR, Gundry CR, Heithoff KB. Lumbar disc high-intensity zone. Correlation of magnetic resonance imaging and discography. Spine 1996; 21:79-86.

122. Schellhas KP, Smith MD, Gundry CR, Pollei SR. Cervical discogenic pain. Prospective correlation of magnetic resonance imaging and discography in asymptomatic subjects and pain sufferers. Spine 1996; 21:300-311.

123. Schellhas KP, Garvey TA, Johnson BA, Rothbart PJ, Pollei SR. Cervical diskog raphy: analysis of provoked responses at $\mathrm{C}_{2}-\mathrm{C}_{3}, \mathrm{C}_{3}-\mathrm{C}_{4}$, and $\mathrm{C}_{4}-\mathrm{C}_{5}$. AJNR Am J Neuroradiol 2000; 21:269-275.

124. Schellhas KP, Pollei SR, Dorwart RH. Thoracic discography. A safe and reliable technique. Spine 1994; 19:21032109.

125. Schwarzer AC, Aprill CN, Derby R, Fortin J, Kine G, Bogduk N. The prevalence and clinical features of internal disc disruption in patients with chronic low back pain. Spine 1995; 20:1878-1883.

126. Schwarzer AC, Aprill CN, Derby R, Fortin J, Kine G, Bogduk N. The relative contributions of the disc and zygapophyseal joint in chronic low back pain. Spine 1994; 19:801-806.

127. Siebenrock KA, Aebi M. Cervical discography in discogenic pain syndrome and its predictive value for cervical fusion. Arch Orthop Trauma Surg 1994; 113:199-203.

128. Simmons JW, Emery SF, McMillin JN, Landa D, Kimmich SJ. Awake discography. A comparison study with magnetic resonance imaging. Spine 1991; 16: S216-S221.

129. Smith BM, Hurwitz EL, Solsberg D, Rubinstein D, Corenman DS, Dwyer AP, Kleiner J. Interobserver reliability of detecting lumbar intervertebral disc high- intensity zone on magnetic resonance imaging and association of high-intensity zone with pain and anular disruption. Spine 1998; 23:2074-2080.

130. Smith SE, Darden BV, Rhyne AL, Wood KE. Outcome of unoperated discogram-positive low back pain. Spine 1995; 20:1997-2000.

131. Vanharanta H, Sachs BL, Ohnmeiss DD, Aprill C, Spivey M, Guyer RD, Rashbaum RF, Hochschuler SH, Terry A, Selby D, Stith WJ,. Mooney V. Pain provocation and disc deterioration by age. A CT/discography study in a low-back pain population. Spine 1989; 14:420-423.

132. Vanharanta H, Sachs BL, Spivey M, Hochschuler SH, Guyer RD, Rashbaum RF, Ohnmeiss DD, Mooney V. A comparison of $\mathrm{CT} /$ discography, pain response and radiographic disc height. Spine 1988; 13:321-324.

133. Vanharanta H, Guyer RD, Ohnmeiss DD, Stith WJ, Sachs BL, Aprill C. Spivey M, Rashbaum RF, Hochschuler SH, Videman T, Selby DK, Terry A, Mooney V. Disc deterioration in low-back syndromes. A prospective, multi-center CT/discography study. Spine 1988; 13:1349-1351.

134. Weishaupt D, Zanetti M, Hodler J, Min $\mathrm{K}$, Fuchs B, Pfirrmann CW, Boos N. Painful lumbar disk derangement: relevance of endplate abnormalities at MR imaging. Radiology 2001; 218:420427.

135. Walsh TR, Weinstein JN, Spratt KF, Lehmann TR, Aprill C, Sayre H. Lumbar discography in normal subjects. A controlled, prospective study. J Bone Joint Surg Am 1990; 72:1081-1088.

136. Wetzel FT, LaRocca SH, Lowery GL, Aprill $\mathrm{CN}$. The treatment of lumbar spinal pain syndromes diagnosed by discography. Lumbar arthrodesis. Spine 1994; 19:792-800.

137. Wood KB, Schellhas KP, Garvey TA, Aeppli $D$. Thoracic discography in healthy individuals. A controlled prospective study of magnetic resonance imaging and discography in asymptomatic and symptomatic individuals. Spine 1999; 24:1548-1555.

138. Yrjama M, Tervonen O, Vanharanta H. Ultrasonic imaging of lumbar discs combined with vibration pain provocation compared with discography in the diagnosis of internal anular fissures of the lumbar spine. Spine 1996; 21:571575 .

139. Zheng Y, Liew SM, Simmons ED. Value of magnetic resonance imaging and discography in determining the level of 
cervical discectomy and fusion. Spine 2004; 29: 2140-2145.

140. Zucherman J, Derby R, Hsu K, Picetti G, Kaiser J, Schofferman J, Goldthwaite N, White A. Normal magnetic resonance imaging with abnormal discography. Spine 1988; 13:1355-1359.

141. Deeks JJ. Systematic reviews in health care: Systematic reviews of evaluations of diagnostic and screening tests. BMJ 2001; 323:157-162.

142. Knottnerus JA, van Weel C, Muris JW. Evaluation of diagnostic procedures. $B M J$ 2002; 324:477-480.

143. Bahado-Singh RO, Oz AU, Kovanci E,
Deren O, Copel J, Baumgarten A, Mahoney J. New Down syndrome screening algorithm: Ultrasonographic biometry and multiple serum markers combined with maternal age. Am J Obstet Gynecol 1998; 179:1627-1631.

144. Bahado-Singh RO, Oz U, Kovanci E, Cermik D, Flores D, Copel J, Mahoney M, Cole L. New triple screen test for Down syndrome: Combined urine analytes and serum AFP. J Matern Fetal Med 1998; 7:111-114.

145. Wassertheil-Smoller S. Mostly about screening. In Wassertheil-Smoller S (ed). Biostatistics and Epidemiology. A Primer for Health and Biomedical Professionals. Springer-Verlag, New York, 2004, pp 129-140.

146. Weinstein S, Obuchowski NA, Lieber ML. Clinical evaluation of diagnostic tests. AJR Am J Roentgenol 2005; 184:14-19.

147. Riegelman RK. Testing a test (Section II). In Riegelmann RK (ed). Studying a Study and Testing a Test: How to Read the Medical Evidence, 5th Ed. Lippincott Williams \& Wilkins, Philadelphia, 2005, pp 137-192. 